\title{
R7 VU Born-Assessed Demo Plan
}

\author{
Robert Nourgaliev \\ Ralph Nelson
}

February 2010

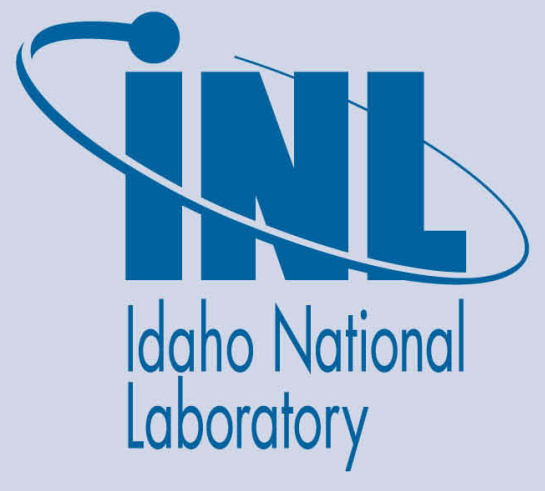

The INL is a U.S. Department of Energy National Laboratory operated by Battelle Energy Alliance 
INL/EXT-10-17979

\title{
R7 VU Born-Assessed Demo Plan
}

\author{
Robert Nourgaliev \\ Ralph Nelson
}

February 2010

\section{Idaho National Laboratory \\ Idaho Falls, Idaho 83415}

http://www.inl.gov

\author{
Prepared for the \\ U.S. Department of Energy \\ Office of Nuclear Energy \\ Under DOE Idaho Operations Office \\ Contract DE-AC07-05ID14517
}




\section{R7 VU Born-Assessed Demo Plan}

by

Robert Nourgaliev

Ralph Nelson

\section{Introduction}

This is an initial draft of a born-assessed VU plan for the RELAP7 (R7) code development effort. The plan will continue to evolve based on the growth of code capability. This growth will be reflected as additional testing is identified and done. Later versions of this document will reflect that growth.

\section{VR-Demo}

To demonstrate the born-assessed VU task, we will build the R7 demonstration around the model of the Virtual-Reactor \#1 (VR1) problem, Figure 1. This is a simple thermalhydraulics loop, made of 16 components, including core, pump, heat exchanger and pressurizer. This single-phase water $\mathrm{T} / \mathrm{H}$ loop enables testing core cooling using both forced and natural circulation - a representative of the R7 code ability to model future passive reactor safety systems.

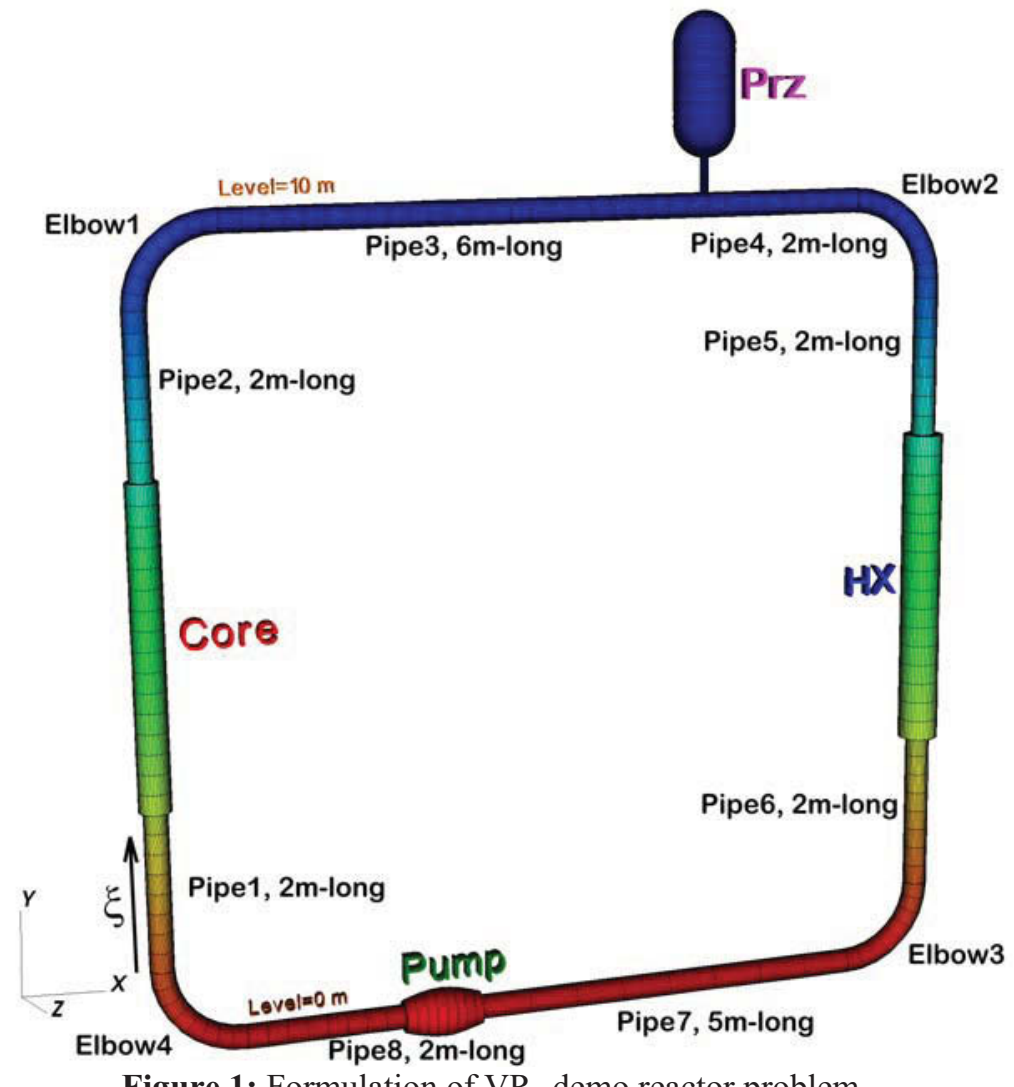

Figure 1: Formulation of $\mathrm{VR}_{1}$ demo reactor problem.

A simple control system will be designed to explore system response to different accident scenarios, with ability to couple with probability risk analysis (PRA), a study separate from this born-assessed effort. 


\section{Verification}

\section{Long Term Project Goals}

The born-assessed verification effort shall provide the following:

○ Document any verification techniques used and any domain of verification (e.g., the conditions under which verification was conducted).

○ Document any numerical error estimates (e.g., numerical approximations, insufficient discretization, insufficient iterative convergence, finite-precision arithmetic, space-time convergence estimated using exact/manufactured solutions and Richardson extrapolations) for the results of the computational model.

- Document the verification status of (conceptual, mathematical, and computational) models.

- Quantify the uncertainties present in the numerics used and propagate them into application predictions.

\section{First Year Deliverables}

All the above shall be done for each component of the system, i.e. Pipe, Elbow, Pump, Pressurizer, Core and Heat Exchanger, designing challenging manufactured solutions for each.

\section{Validation}

\section{Long Term Project Goals}

The born-assessed validation effort shall provide the following:

○ Document any techniques used to validate the M\&S for its intended use, including the experimental design and analysis, and the domain of validation.

- Document any validation metrics, referents, and data sets used for model validation.

- Document any studies conducted and results of model validation.

- Quantify the uncertainties present in model validation with comparisons to data.

- Identify major sensitivities within models to enable model development or further experimental activities that could reduce predictive uncertainties based on best investment strategies.

\section{First Year Deliverables}

One challenge associated with the use of legacy data in many validation efforts is the lack of replicate data (repeated tests) to identify the aleatory nature of such tests. This limits the full range of UQ validation testing that can be done.

As a first step, we will demonstrate validation for wall friction in the case of water flowing inside smooth and rough (industrial) pipes. This will include direct comparison with available relevant experimental data. Validation of this capability is relevant to the VR1 model for the PRA related study noted above. 


\section{Uncertainty Quantification (UQ)}

Uncertainty quantification is the foundation of a modern VU effort. All other elements of the effort are built upon it as the foundation. Identification of the major uncertainties in any prediction is one of its key outcomes. This identification allows the VU team to develop investment strategies that can be used to minimize those uncertainties as needed for design or licensing purposes.

\section{Long Term Project Goals}

The born-assessed UQ effort shall provide the following:

$\circ$ Document any uncertainty quantification processes used for

a. The reference data.

b. The input data.

c. The M\&S results.

d. The propagation of uncertainties.

e. The quantities derived from $M \& S$ results.

- Document any quantified uncertainties, both physical and numerical, for

a. The reference data.

b. The input data.

c. The M\&S results.

d. The propagation of uncertainties.

e. The quantities derived from $M \& S$ results.

- Document the extent and results of any sensitivity analyses performed with the $\mathrm{M} \& \mathrm{~S}$.

a. Any aspects of M\&S that have not been verified.

b. Any aspects of M\&S that have not been validated.

c. If any significant physical processes, effects, scenarios, or environments have not been considered in the uncertainty quantification analysis.

\section{First Year Deliverables}

As a first step, we will couple R7 with SNL Dakota code, demonstrating Dakota's capabilities to determine model variable sensitivities applied to VR1 demo reactor. Sensitivity studies are a preliminary step toward UQ and represent a measure of variable importance based on expert estimates of variable ranges.

\section{Calibration}

\section{Long Term Project Goals}

Calibration done under the born-assessed effort shall provide the following:

- Document calibration processes used.

○ Document before and after results.

\section{First Year Deliverables}


It should also be noted that the lack of aleatory characteristics in much of the legacy data also affects the nature of the calibration that can be done.

Identification of calibration tasks is not yet possible and will be defined as the project proceeds. 